\title{
Risk factors for perioperative blood transfusions after urogenital fistula repair in Uganda: a retrospective cohort study
}

\author{
Thrisha Potluri ${ }^{1}$, Lauren Holt ${ }^{1}$, Jean Paul Tanner ${ }^{2}$, Lucien Wasingya ${ }^{3}$, Shane Duffy ${ }^{4}$, and \\ Kristie Greene ${ }^{1}$ \\ ${ }^{1}$ University of South Florida College of Medicine \\ ${ }^{2}$ University of South Florida College of Public Health \\ ${ }^{3}$ Kitovu Hospital \\ ${ }^{4}$ Chelsea and Westminster Hospital
}

February 28, 2021

\begin{abstract}
Objective: To determine the incidence of and risk factors for perioperative blood transfusions after urogenital fistula repairs in Uganda. Design: A retrospective cohort study. Setting: A community hospital in Masaka, Uganda. Population: Women who underwent fistula repair at the Kitovu Hospital between 2013 and 2019. Methods: Retrospective review of demographics, and clinical perioperative characteristics of patients surgically treated for urogenital fistula. Patient characteristics were compared between those who did and did not require a blood transfusion. Main Outcome Measures: need for perioperative blood transfusion, risk factors Results: 546 patients treated for urogenital fistulas were included in this study. The median age was $31.1 \pm 13.2$. A vaginal surgical approach was used in the majority of patients (84.6\%). Complications occurred in $3.5 \%$ of surgical repairs, and the incidence of blood transfusions was $6.2 \%$. In multivariable analyses, women with fistula repairs approached abdominally were 4.3 (95\% CI: 1.85-10.00) times more likely to require transfusions than vaginal operations. A borderline association was observed between timing of repair and perioperative transfusions such that patients who underwent repair after three months from the time of developing the fistula were at lower risk of perioperative transfusions (aOR: 0.48, 95\% CI: 0.22-1.04). Conclusions: The incidence of blood transfusions among urogenital fistula repairs in our population is twice that of developed nations. An abdominal surgical approach to urogenital fistula is a significant risk factor for perioperative blood transfusions. Timing of repair may warrant further study.
\end{abstract}

\section{Introduction:}

It is estimated that more than 2 million young women currently live with untreated obstetric fistulas in sub-Saharan Africa and Asia. There are about 50,000 to 100,000 new cases per year, and approximately 150,000 women in Uganda alone are currently living with this condition. ${ }^{1-3}$ Although surgical fistula repairs are commonly preformed and relatively safe, some of the complications include bowel obstruction, fistula recurrence, and urinary incontinence. ${ }^{4,5}$. A rare complication of fistula surgery is hemorrhaging, accounting for $3 \%$ of all complications. ${ }^{6}$ However, this can be serious and life-threating as access to safe blood for women in low-resource settings can be challenging. Therefore, it is important to understand potential risk factors for perioperative transfusions to allow for proper planning and risk mitigation among high-risk women. There is currently a paucity of information in the literature on this topic. Therefore, we aim to identify the incidence of and risk factors for perioperative blood transfusions after urogenital fistula repair at a Fistula Hospital in Uganda. We hypothesize there will be specific patient characteristics that will increase risk of perioperative transfusions such as location of fistula and route of surgery.

Methods: 
An IRB approved retrospective cohort study was performed to identify the incidence of and risk factors for perioperative blood transfusions after surgical repair of urogenital fistula in Uganda. The study was conducted among women who underwent fistula repair between 2013-2019 at the Kitovu Hospital in Masaka, Uganda. Kitovu Hospital is one of three private, faith-based hospitals under the partnership of the USAID Fistula Care Plus. Complex fistula cases from many geographical areas are often referred to the hospital which has a specialized fistula repair unit. All types of urogenital fistula were included (vesicovaginal, urethrovaginal, vesicouterine, and those with ureteral involvement). Those who had concomitant rectovaginal fistulas were included so long as they underwent repair of the urogenital fistula. A surgical database, established in 2013 from patient charts, was used to identify those who underwent urogenital fistula repair during that time period and determine eligibility for inclusion. After identification of included patients within our cohort, a retrospective chart review was performed to gather all remaining variables of interest including patient demographics, medical and surgical history, as well as perioperative details. Primary outcome was the need for perioperative blood transfusion. Patient characteristics were then compared between those who did and did not require perioperative blood transfusion after urogenital fistula repair.

Descriptive statistics were calculated for a number of demographic and clinical characteristics to describe the full sample and women with and without a transfusion. Results were reported as mean with standard deviation (or median with interquartile range) for continuous variables and frequency with percentages for categorical variables. Logistic regression was used to estimate unadjusted odds ratios (ORs) and $95 \%$ confidence intervals (CIs) representing the association between each risk factor of interest and blood transfusion. Risk factors were chosen a priori based on previous literature and clinical knowledge and included patient age, time with fistula, type of delivery, delivery outcome, cause of fistula, history of fistula repair, HIV status, presence of foot drop, surgical approach, anesthesia type, concomitant surgery, use of a graft or flap as part of their surgery, a concomitant sling procedure, complications, use of ureteric catheter, and surgeon training. A multivariable logistic regression model was used to estimate adjusted ORs and 95\% CIs; only covariates that were significantly associated with risk of blood transfusion in univariate analyses were included in the adjusted model. Categorical subgroups were created for covariates with missing data and included in the models. All statistical tests were two-sided and declared significant at $\mathrm{p}<0.05$. Statistical analyses were performed with SAS software, version 9.4 (SAS Institute, Inc., Cary, NC).

\section{Results:}

A total of 546 patients who underwent fistula repairs from 2013-2019 met inclusion criteria and were included in the study. Patient characteristics are outlined in Table 1 . The mean age was $31.3 \pm 13.2$. Median duration of labor was 48 hours (interquartile range: $36-72$ hours). Median parity was 3 (interquartile range: 1-6). The median time suffering from the condition before repair was 6 months (interquartile range: $0.25-5$ years). Preoperative characteristics are described in Table 2. Most fistula patients delivered via Cesarean section $(58.6 \%)$ and $23.8 \%$ delivered via spontaneous vaginal delivery. A majority of fistula patients delivered still births (65.4) versus livebirths (17.6\%). A quarter of patients had undergone previous repair of their fistula (25.5\%). The most common location of fistulas was juxta-cervical or vault fistulas (20.7\%). A vaginal surgical approach was used in the majority of patients (84.6\%) compared to an abdominal approach (11.7\%). Postoperative complications occurred in $3.5 \%$ of patients. The incidence rate of blood transfusions was $6.2 \%$.

Risk factors examined are included in Table 3 . The risk factors that were associated with blood transfusions in univariate analyses were time with fistula, surgical approach, anesthesia type, and whether the patient had any complications. Multivariable analyses identified abdominal surgical approach as the only statistically significant risk factor of a need for blood transfusions. Surgical fistula repairs approached abdominally (non-vaginally) were four times more likely (aOR: 4.30, 95\% CI: 1.85-10.00) to require transfusions than vaginal operations. An inverse borderline association was observed between timing of repair and perioperative transfusions, such that patients who underwent repair after three months from the time of developing the fistula were at lower risk of perioperative transfusions (aOR: 0.48, 95\% CI: 0.22-1.04).

\section{Discussion:}


Main Findings: This retrospective study examines risk factors for patients who underwent surgical repair of urogenital fistulas at a Fistula Hospital in Masaka, Uganda. The data demonstrate that the incidence rate of blood transfusions in our patient population is $6.2 \%$. Consistent with our hypothesis, there are specific patient characteristics that predict a need for perioperative blood transfusions. Specifically, these risk factors include abdominal approach and timing of fistula repair was borderline.

Strengths and Limitations: Strengths of this study include well-defined outcomes and their importance given the lack of availability of blood in many areas of the world. This is the first study to our knowledge looking at this important outcome. Furthermore, we include a population that is generalizable to obstetric fistula populations in the developing world. The surgeries were completed by a group of well-trained fistula surgeons from both Uganda as well as other countries who worked collaboratively to care for these women in a dedicated Fistula Hospital. Limitations include a retrospective nature of this study and therefore missing data in some cases that prevent us from looking at other risk factors that may not have been collected at the time or even known by the patients themselves. Some of these include other patient comorbidities or history of previous non-fistula surgeries for example.

Interpretation: Fistulas approached abdominally were approximately four time more likely to need a blood transfusion compared to a vaginal approach. This is consistent with existing research advocating for vaginal approach as the preferred management option for surgical fistula repairs. ${ }^{7}$ A vaginal approach is typically preferred when feasible due to its minimally invasive nature and association with shorter operative times, decreased blood loss and shorter length of stays in hospitals. ${ }^{8,9}$ Previous studies have demonstrated higher rate of complications associated with an abdominal approach, including urinary tract infections, sepsis, blood transfusions, and hospital readmissions. ${ }^{6,7}$ Results from our study are consistent with published data advocating for vaginal approach in regards to risk of perioperative blood transfusion as well. However, there are circumstances in which an abdominal approach may be indicated, such as for ureteric involvement, limited vaginal access, significant associated scarring or failed prior repairs. ${ }^{7,8,10}$ Ultimately, approach is largely determined by patient characteristics, size and location of fistula and expertise of surgeon. Although an abdominal approach may be unavoidable at times, our data may help surgeons identify these higher risk patients in preoperative surgical planning and ensuring availability of resources such as sufficient blood.

In our study, timing of repair was also identified as a borderline risk factor with patients who underwent fistula repair before three months being approximately half as likely to receive a blood transfusion. Although this was a borderline relationship in our study, the authors feel it is notable and may require future studies to fully investigate. Timing of repair is often left to the surgeon's discretion, based on evaluation of the tissue quality and maturity of the fistula. Traditional teaching typically dictates waiting at least three months after childbirth before surgically repairing a fistula to allow time for healing and inflammation to subside as well as recovery from central necrosis, edema, or infection. ${ }^{4,7,11}$ Additionally, conservative management for women who present within the first three months for repair may help the fistula spontaneously close. ${ }^{4}$ However, some experts would argue, that it is reasonable to repair before the 3-month mark so long as the fistula tract is mature and the tissue appears healthy. Furthermore, studies have demonstrated earlier closures within 1-2 weeks of injury may have similar success rates to delayed repairs. ${ }^{8,12,13}$ Our results suggest that there may be additional benefits to delayed repair in regards to decreasing associated risk for perioperative blood transfusions particularly in low resource settings where blood may not be readily available.

In our study, timing of repair was also identified as a borderline risk factor with patients who underwent fistula repair before three months being approximately half as likely to receive a blood transfusion. Although this was a borderline relationship in our study, the authors feel it is notable and may require future studies to fully investigate. Timing of repair is often left to the surgeon's discretion, based on evaluation of the tissue quality and maturity of the fistula. Traditional teaching typically dictates waiting at least three months after childbirth before surgically repairing a fistula to allow time for healing and inflammation to subside as well as recovery from central necrosis, edema, or infection. ${ }^{4,7,11}$ Additionally, conservative management for women who present within the first three months for repair may help the fistula spontaneously close. ${ }^{4}$ However, some experts would argue, that it is reasonable to repair before the 3 -month mark so long as the fistula tract 
is mature and the tissue appears healthy. Furthermore, studies have demonstrated earlier closures within 1-2 weeks of injury may have similar success rates to delayed repairs. ${ }^{8,12,13}$ Our results suggest that there may be additional benefits to delayed repair in regards to decreasing associated risk for perioperative blood transfusions particularly in low resource settings where blood may not be readily available.

Adequately powered prospective case-control studies would provide the best estimates of risk factors and potentially identify other characteristics that serve as risk factors for needing a perioperative blood transfusion, but such trials would require a large sample size. In the interim, we believe our study represents important evidence for consideration in guiding surgical practice in low-resource settings.

\section{Conclusion:}

In conclusion, risk of perioperative blood transfusions was approximately $6 \%$. Women whose fistulas were approached abdominally were at higher risk for requiring perioperative blood transfusion. The borderline relationship between timing of fistula repair and blood transfusion may warrant further study. Identification of patients who may be at higher risk for requiring blood transfusions may help in preoperative planning in low resource settings.

\section{Acknowledgements:}

We are grateful to the international fistula surgeons, doctors, nurses and staff at Kitovu Health Care Complex in Masaka, Uganda that helped provide and collect patient data as well as the Chelsea and Westminster Global Health Fellows for their considerable contributions in starting this surgical database. Kitovu staff and the Fellows have no conflict of interest in relation to our data analysis and manuscript preparation.

Disclosure of Interests: The authors report no conflicts of interest.

\section{Contribution to Authorship:}

TKP was involved in conception, planning, carrying out, analyzing and writing up this work. LMH was involved in carrying out the data collection of this study. JPT was involved with analyzing and writing up this work. SD was involved with conception of this study. LW was involved with conception of this study and carrying out data collection. KAG was involved with conception, planning, analyzing and writing up this work.

Details of Ethics Approval: As this research did not use methods involving human subjects, it was deemed exempt from IRB approval and oversight on 04/05/2019 by the USF Institutional Review Board at the University of South Florida's Human Research Protection Program. (IRB\#: Pro00039874)

Funding: This research did not receive any specific grant from funding agencies in the public, commercial, or not-for-profit sectors.

\section{References:}

1. World Health Organization. (2019). 10 facts on obstetric fistula . [online] Available at: https://www.who.int/features/factfiles/obstetric_fistula/en/ [Accessed 11 Mar. 2019].

2. Maheu-Giroux M, Filippi V, Samadoulougou S et al. Prevalence of symptoms of vaginal fistula in 19 sub-Saharan Africa countries: a meta-analysis of national household survey data. Lancet Glob Health. 2015. 3(5):e271-8. doi: 10.1016/S2214-109X(14)70348-1.

3. ICF International: The DHS Program. (2016). Uganda Dempgraphic and Health Survey 2016: Key indicators report. Available at: https://dhsprogram.com/pubs/pdf/FR333/FR333.pdf [Accessed 22 Aug. 2019]

4. Wall LL. Obstetric vesicovaginal fistula as an international public-health problem. Lancet. 2006. 268(9542):1201-9.

5. Shefren JM. The tragedy of obstetric fistula and strategies for prevention. Am J Obstet Gynecol. 2009; 200(6):668-71. 
6. Theofanides MC, Sui W, Sebesta EM et al. Vesicovaginal fistulas in the developed world: an analysis of disease characteristics, treatments, and complications of surgical repair using the ACS-NSQIP database. Neurourol Urodyn. 2017. 36(6):1622-28.

7. Lee D, Zimmern P. Vaginal approach to vesicovaginal fistula. Urol Clin North Am. 2019. 46(1):123-33. doi: 10.1016/j.ucl.2018.08.010.

8. McKay E, Watts K, Abraham N. Abdominal approach to vesicovaginal fistula. Urol Clin N Am. 2019; 46(1):135-46.

9. Garely AD, Mann WJ. Urogenital tract fistulas in women. UpToDate. 2018.

10. Michael S, Baggish MMMK. Atlas of pelvic anatomy and gynecologic surgery. Elsevier Saunders. 2006.

11. Malik MA, Sohail M, Malik MTB et al. Changing trends in the etiology and management of vesicovaginal fistula. Int J Urol. 2018. 25(1):25-9. doi: 10.1111/iju.13419.

12. Singh O, Gupta SS, Mathur RK. Urogenital fistulas in women: 5-year experience at a single center. Urol J. 2010. 7(1):35-9.

13. Hadley HR. Vesicocaginal fistula. Curr Urol Rep. 2002. 3(5):401-7.

14. Hillary CJ, Osman NI, Chapple CR. The aetiology, treatment, and outcome of urogenital fistulae managed in well- and low-resourced countries: a systematic review. Eur Urol. 2016. 70(3):478-92.

15. Sori DA, Azale AW, Gemeda DH. Charactertics and repair outcome of patients with vesicovaginal fistula managed in Jimma University teaching hospital, Ethiopia. BMC Urology. 2016. 16(1):41. doi: 10.1186/s12894-016-0152-8.

16. Breen M, Ingber M. Controversies in the management of vesicovaginal fistula. Best Pract Res Clin Obstet Gynaecol. 2019. 54:61-72.

17. Frajzyngier V, Ruminjo J, Asiimwe F et al. Factors influencing choice of surgical route of repair of genitourinary fistula, and the influence of route of repair on surgical outcomes: findings from a prospective cohort study. BJOG. 2012. 119(11):1344-53.

18. Frajzyngier V, Ruminjo J, Barone MA. Factors influencing urinary fistula repair outcomes in developing countries: a systematic review. Am J Obstet Gynecol. 2012. 207(4):248-58.

19. Goh JT, Browning A, Berhan B, Chang A. Predicting the risk of failure of closure of obstetric fistula and residual urinary incontinence using a classification system. Int Urogynecol J Pelvic Floor Dysfunct. 2008. 19(12):1659-62

20. Nardos R, Browning A, Chen CC. Risk factors that predict failure after vaginal repair of obstetric vesicovaginal fistulae. Am J Obstet Gynecol. 2009. 200(5):578.e1-4.

21. Hawkins L, Spitzer RF, Christoffersen-Deb A, Leah J, Mabeya H. Characteristics and surgical success of patients presenting for repair of obstetric fistula in western Kenya. Int J Gynaecol Obstet. 2013. 120(2):178-82. doi: 10.1016/j.ijgo.2012.08.014.

\section{Hosted file}

VVFS_Blood RFs_Tables_BJOG.pdf available at https://authorea.com/users/398675/articles/ 511304-risk-factors-for-perioperative-blood-transfusions-after-urogenital-fistularepair-in-uganda-a-retrospective-cohort-study 